\title{
ORIGINAL
}

\section{EVOLUCIÓN DE LA MORTALIDAD POR CÁNCER DE MAMA Y DISEMINACIÓN DE LA MAMOGRAFÍA DE CRIBADO EN CATALUÑA. UN ANÁLISIS POR REGIONES SANITARIAS}

\author{
María José Pérez Lacasta (1), Aleix Gregori Gomis (1), Misericòrdia Carles Lavila (1), Rosa Gispert \\ Magarolas (2), Montserrat Martínez Alonso (3,4), Ester Vilaprinyo Terré (5), Roger Pla Farnós (6) y \\ Montserrat Rué Monné $(3,4)$ \\ (1) Departament d'Economia, Universitat Rovira i Virgili. \\ (2) Servei d'Informació i Estudis, Departament de Salut, Generalitat de Cataluña. \\ (3) Institut de Recerca Biomèdica de Lleida (IRBLleida), Lleida. \\ (4) Departament de Ciències Mèdiques Bàsiques, Facultat de Medicina, Universitat de Lleida. \\ (5) Servei d'Avaluació i Epidemiologia Clínica, IMIM-Hospital del Mar, Barcelona. \\ (6) Gerència Territorial de l'Institut Català de la Salut a Terres de l'Ebre, Tortosa.
}

\section{RESUMEN}

Fundamento: El descenso de las tasas de mortalidad por cáncer de mama (CM) se ha atribuido a la implantación de programas de cribado y a avances terapéuticos. El objetivo de este trabajo es comparar la evolución de su mortalidad en las regiones sanitarias de Cataluña en el periodo 19932007. Paralelamente, se ha analizado la diseminación de la mamografía periódica en las regiones sanitarias.

Métodos: Se analizaron los datos del registro de mortalidad y encuestas de salud. Se utilizaron regresiones de Poisson y «joinpoint» para comparar las tasas de mortalidad por CM y analizar su evolución temporal. Se utilizaron modelos de efectos mixtos para comparar el nivel y la evolución de la mortalidad por regiones.

Resultados. La tasa de mortalidad por CM descendió un 3\% anual en Cataluña. Entre 1993 y 2007, la tasa estandarizada varió de 34,8 a 23,3 por 100.000 mujeres. Barcelona ciutat presentó unas tasas de mortalidad más elevadas que las regiones Centre (ratio de tasas $(\mathrm{RT})=0,87$ ), Costa de Ponent $(\mathrm{RT}=0,89)$, Tarragona $(\mathrm{RT}=0,9)$ y Lleida $(\mathrm{RT}=0,915)$, pero estas diferencias tendieron a desaparecer. No se observaron cambios de tendencia en la evolución de la mortalidad de las regiones, excepto en la región Centre. Durante los años 1990 Barcelona ciutat presentó unos porcentajes de utilización de mamografia periódica del $36,1 \%$ de las mujeres de $40-74$ años, en la encuesta de 1994 , la región Centre $(23,7 \%)$ y Costa de Ponent $(25,2 \%)$.

Conclusiones: La progresiva utilización de mamografía periódica y la disminución de la mortalidad por CM fueron similares en las regiones sanitarias de Cataluña.

Palabras clave: Cáncer de mama. Mortalidad. Detección precoz. Tendencias.

Correspondencia:

Montserrat Rué

Departament de Ciències Mèdiques Bàsiques, Facultat de Medicina, Universitat de Lleida

C/ Montserrat Roig 2, 25008 Lleida

montse.rue@cmb.udl.cat

Financiación: Este estudio ha recibido financiación del Fondo de Investigación Sanitaria (proyectos PI06/1649 y PI06/90355) y de l'Agència d'Avaluació de Tecnologia i Recerca Mèdiques de la Generalitat de Cataluña (proyecto 068/27/06).

\section{ABSTRACT}

\section{The Evolution of Breast Cancer Mortality and the Dissemination of Mammography in Catalonia. An Analysis by Health Region}

Background: The decrease of breast cancer (BC) mortality rates has been attributed to early detection programs and therapeutic advances. The objective is to compare $\mathrm{BC}$ mortality trend in health regions of Catalonia during the period 1993-2007. In parallel, dissemination of periodic mammography in the health regions has been analyzed.

Methods: Mortality and health surveys data were used. Poisson and «joinpoint» regression analyses were used to compare regional BC mortality rates and quantify their temporal evolution. Mixed effects models were used to compare the rates and their evolution by region.

Results: The BC mortality rate decreased 3\% annually in Cataluña. Between 1993 and 2007, the standard mortality rate changed from 34.8 to 23.3 per 100,000 women. Barcelona ciutat showed higher mortality rates than the Centre (rate ratio $(\mathrm{RR})=0.873)$, Costa de Ponent $(\mathrm{RR}=0.885)$, Tarragona $(\mathrm{RR}=0.9)$ and Lleida regions $(\mathrm{RR}=0.915)$, but these differences tend to disappear over time. There were no observed trend changes in the evolution of the regional mortality rates, except in the Centre region. The use of periodic mammography was similar across health regions. During the 90 s, Barcelona ciutat had a $36.1 \%$ utilization of periodic mammography in women aged 40-74, in the 1994 survey, the Centre 23.7 and Costa de Ponent $25.2 \%$.

Conclusions: The progressive increase in the use of periodic mammography and the decrease of BC mortality were similar in the eight health regions of Catalonia.

Key words: Breast cancer. Mortality. Early detection of cancer. Trends.

Conflictos de intereses: Los autores declaran no tener conflictos de intereses. 


\section{INTRODUCCIÓN}

El cáncer de mama (CM) es uno de los tumores con mayor incidencia entre la población femenina en todo el mundo ${ }^{1}$. El descenso de las tasas de mortalidad por CM en los países occidentales desde la década de los noventa se ha atribuido en gran parte a la implantación de programas de cribado y a los avances terapéuticos ${ }^{2}$.

En Cataluña, la mortalidad por CM aumentó un $1,5 \%$ anual hasta principios de la década de los noventa y luego pasó a disminuir por razón del $2,7 \%$ anual ${ }^{3}$. Este declive de la mortalidad se observó en todos los grupos de edad, aunque se inició más tarde en mujeres mayores ${ }^{4,5}$. Se estima que se mantendrá una tendencia decreciente de la mortalidad por CM en los próximos años y en todos los grupos de edad ${ }^{6}$.

La reducción de la mortalidad por CM pasa por tres medidas estratégicas: la identificación de grupos de riesgo, la detección precoz y la efectividad de los tratamientos. Las políticas sanitarias públicas de prevención generalmente inciden en las dos primeras, identificando el grupo diana e incentivando la utilización por parte de las mujeres de la mamografía de cribado ${ }^{7}$. La población diana se define por la edad de la mujer y por la demostración en estudios aleatorizados de que el uso de la mamografía ha sido efectivo en reducir la mortalidad. En Cataluña, el programa de detección precoz de CM comenzó en el año 1992, con una prueba piloto llevada a cabo en Molins de Rei y Sant Feliu de Llobregat, en la región sanitaria Costa de Ponent. A partir de 1995 y hasta 2002 se fue incorporando progresivamente el resto del territorio, y en el año 2004 el $100 \%$ de la población diana quedó cubierta en todas las regiones sanitarias. Las regiones sanitarias del territorio catalán son áreas delimitadas a partir de factores geográficos, socioeconómicos y demográficos que despliegan su actividad en los ámbitos de la planificación, de la compra de servicios, los sis- temas de gestión y de la atención al cliente. Actualmente, el programa de detección precoz del CM tiene una población diana de mujeres entre 50 y 69 años, con una participación en el mismo del 65\% (Departament de Salut, 2006).

Las Encuestas de Salud de Cataluña (ESCA) de los años 1994 y 2002 mostraron diferencias en la utilización de la mamografía preventiva en las regiones sanitarias cata$\operatorname{lanas}^{8}$. Estas diferencias, asociadas al nivel socioeconómico de las mujeres ${ }^{9}$, o al tipo de población que predomina en cada región, rural o urbana, disminuyeron en el periodo estudiado paralelamente a la diseminación de la mamografía de cribado a través de los programas poblacionales.

El objetivo de este estudio es analizar la mortalidad por CM en las regiones sanitarias de Cataluña, en el periodo 1993-2007, comparando las tasas de mortalidad entre regiones y evaluar si han existido diferencias en su evolución. Paralelamente, se ha analizado la diseminación de la mamografía periódica en las regiones sanitarias.

\section{MATERIAL Y MÉTODOS}

Fuentes de información. Los datos de mortalidad proceden del Registro de Mortalidad del Departament de Salut de la Generalitat de Cataluña y corresponden a todas las defunciones por cáncer de mama de mujeres residentes, que ocurrieron en Cataluña entre los años 1975 y 2007 inclusive. Para el periodo 1993-2007 se dispuso de la región sanitaria de residencia. Los denominadores poblacionales son estimaciones inter y postcensales de los mismos años que se obtuvieron de la base de datos del Institut d'Estadística de Cataluña (IDESCAT) ${ }^{10}$.

A partir de las encuestas de salud de Cataluña de los años 1994, 2002 y 2006 $6^{11-13}$ se obtuvo el porcentaje de mujeres que se realizaron una mamografía periódica, la frecuen- 
cia con que lo hicieron y los motivos, todo ello por grupos de edad. Hubo algunos cambios en la forma de preguntar sobre la utilización de la mamografía, pero en las tres encuestas se preguntó a las mujeres si se hacían mamografías periódicas. En las encuestas de 1994 y 2002 se preguntó si se las hacían de forma preventiva y en la de 2006 si se las hacían a través del programa de detección precoz, del ginecólogo del sistema de salud, de una mutua, etc.

Aunque el mapa sanitario catalán cambió en el año 2005, la encuesta del año 2006 permite obtener también la información en función del mapa antiguo. Dicha agrupación territorial constaba de ocho regiones: Barcelona ciutat, Barcelonès Nord-Maresme, Centre, Costa de Ponent, Girona, Lleida, Tarragona y Tortosa. El año 2007 la región menos poblada, Tortosa, tenía 71.400 mujeres residentes, la región más poblada, Barcelona ciutat, tenía 840.000 y el número medio de mujeres residentes en las ocho regiones era 454.000 .

Mortalidad por cáncer de mama en las regiones sanitarias de Cataluña en el periodo 1993-2007. Las tasas de mortalidad por CM en las regiones se estandarizaron con el método directo tomando como población de referencia la de Cataluña del año 1993. Se utilizó un modelo de regresión de Poisson para comparar la mortalidad por $\mathrm{CM}$ de las ocho regiones, ajustando por edad $^{14}$. Se evaluó si había sobredispersión y en caso afirmativo se ajustó un modelo binomial negativo. Se obtuvieron las ratios de las tasas de mortalidad mediante la transformación exponencial de los coeficientes del modelo. Se tomó como grupo de referencia la región Barcelona ciutat, la más poblada, con el fin de obtener estimaciones más estables. Se compararon los modelos que incluían la edad en forma lineal o categórica mediante el test de cociente de verosimilitudes y se seleccionó el modelo que ajustaba mejor los datos observados.
Se utilizó el análisis de regresión lineal «joinpoint» para evaluar los cambios en la tendencia temporal de las tasas ${ }^{15}$. Es un método que contrasta si uno o más puntos de inflexión son estadísticamente significativos y deben incorporarse al modelo para mejorar el ajuste. También se obtiene una estimación del cambio porcentual anual en cada tramo del modelo mediante un modelo lineal generalizado con distribución de Poisson. Se utilizó el programa «Joinpoint» del Surveillance Research Program del US National Cancer Institute.

Adicionalmente, se decidió utilizar un modelo lineal de efectos aleatorios ${ }^{16}$ para evaluar si había diferencias entre regiones en la pendiente y la ordenada en el origen de las rectas de regresión que describían la evolución temporal de la tasa ajustada de mortalidad por CM. La parte fija del modelo corresponde a la recta de regresión que ajusta la evolución de la mortalidad en el conjunto de Cataluña y los efectos aleatorios representan las desviaciones de los coeficientes de la recta en las regiones respectivas.

Diseminación de la mamografía de cribado en Cataluña y regiones sanitarias. En un trabajo previo se había estudiado la diseminación de la mamografía en Cataluña utilizando curvas logísticas ajustadas mediante modelos de efectos mixtos ${ }^{17}$. El presente estudio se inició con un análisis descriptivo de la utilización (sí/no) y frecuencia (anual, bienal o irregular) de la mamografía periódica en cada región de residencia. A continuación se comparó la proporción de mujeres que utilizan la mamografía periódica en cada región con el valor equivalente obtenido de las curvas de diseminación estimadas para Barcelona ciutat.

Para contrastar la hipótesis de homogeneidad en la diseminación de la mamografía de cribado en las regiones, se utilizaron también modelos de efectos mixtos ${ }^{16}$. Se modeló mediante curvas logísticas el porcentaje de mujeres que utilizaban la mamografía 
periódicamente en función de la edad y la cohorte de nacimiento ${ }^{17}$. Estos modelos incorporaron efectos aleatorios para capturar la variabilidad entre regiones y contrastar la hipótesis de diseminación homogénea entre regiones.

Finalmente se estimaron las odds ratios de utilización de mamografías periódicas en dos grupos de edad, <50 años y 50-69 años, comparando cada región de residencia con Barcelona ciutat. Se utilizó un modelo de regresión cuya variable dependiente era «declara utilizar mamografía de cribado de manera periódica: sí/no» y que incluía la edad y la región sanitaria de residencia como variables explicativas.

Los datos se analizaron mediante los programas Stata/SE $11.0^{18}$, R v. 2.10.1 ${ }^{19}$ y Joinpoint ${ }^{15}$.

\section{RESULTADOS}

Mortalidad por cáncer de mama en Cataluña y regiones sanitarias. La figura 1 muestra la evolución de la tasa estandarizada de mortalidad por CM en Cataluña entre 1975 y 2007. Se observó un porcentaje de

Figura 1

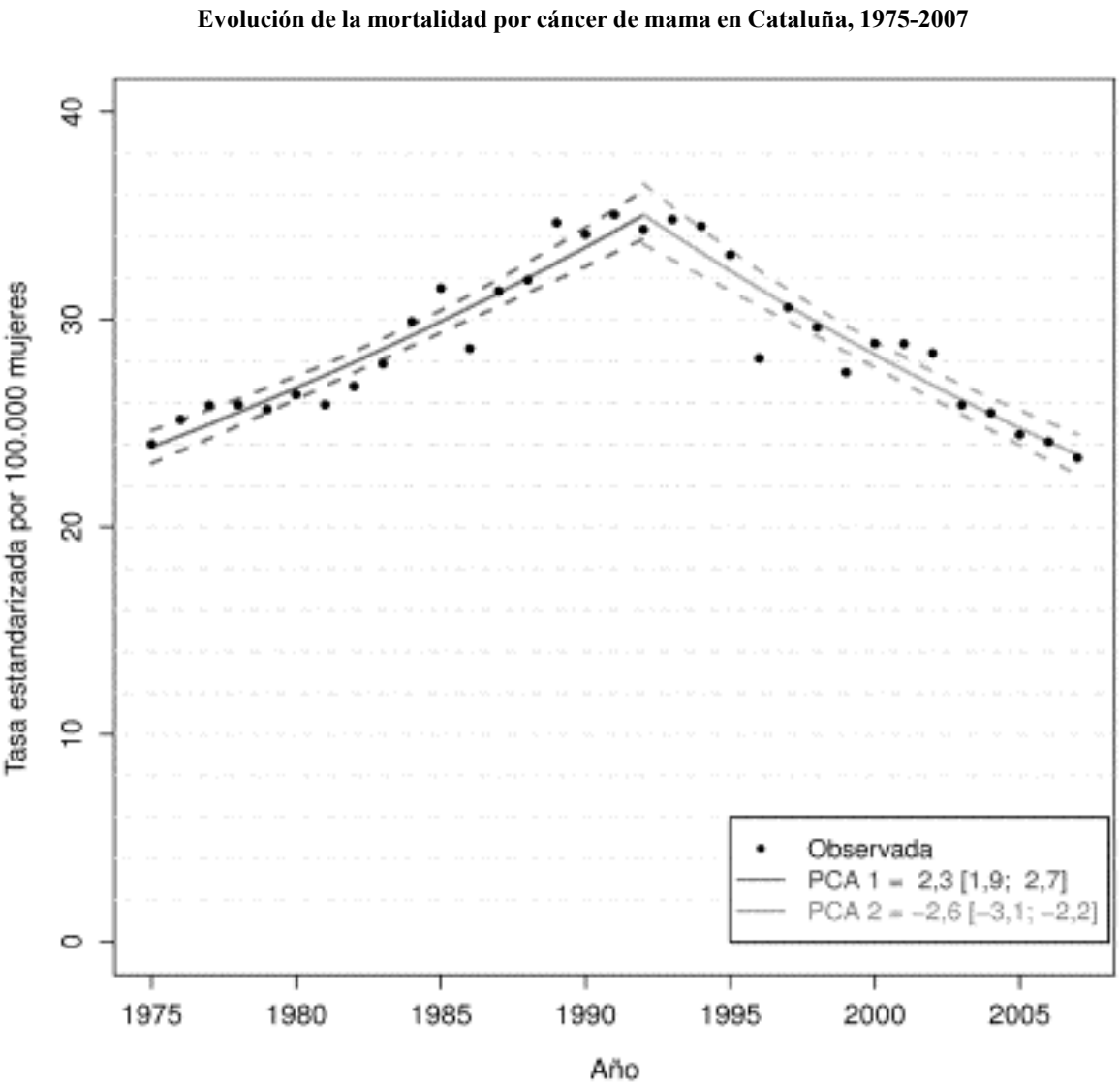

PCA: porcentaje de cambio anual. Tasas estandarizadas según la población de Cataluña de 1993. 
Tabla 1

Modelo de regresión binomial negativa de la mortalidad por cáncer de mama en las regiones sanitarias de Cataluña. (IC: Intervalo de confianza 95\%). Periodo 1993-2007

\begin{tabular}{|l|c|c|c|}
\hline \multicolumn{1}{|c|}{ Variables } & $\begin{array}{c}\text { Ratio de tasas } \\
\text { de mortalidad }\end{array}$ & Valor p & IC 95\% \\
\hline Año (en forma lineal) & 0,97 & $<0,001$ & $0,97-0,98$ \\
\hline Edad (en forma lineal) & 1,06 & $<0,001$ & $1,06-1,06$ \\
\hline Barcelona ciutat (referencia) & 1 & & \\
Barcelonès Nord-Maresme & 0,93 & 0,05 & $0,86-1,00$ \\
Centre & 0,87 & $<0,001$ & $0,82-0,93$ \\
Girona & 0,95 & 0,16 & $0,88-1,02$ \\
Lleida & 0,92 & 0,04 & $0,84-0,99$ \\
Costa de Ponent & 0,89 & $<0,001$ & $0,83-0,95$ \\
Tarragona & 0,90 & 0,01 & $0,83-0,98$ \\
Tortosa & 0,98 & 0,69 & $0,86-1,10$ \\
\hline
\end{tabular}

cambio anual [PCA (Intervalo de confianza $95 \%)$ ] igual a $2,3(1,9-2,7)$ entre 1975 y 1992, seguido de un PCA igual a -2,6 (-3,1; $2,2)$. Esta evolución explica que las tasas ajustadas al final del periodo fueran similares a las del inicio, situándose alrededor de 24 por 100.000 mujeres.

El análisis por regiones sanitarias empezó en 1993, cuando la mortalidad ya había comenzado su tendencia descendente. Entre 1993 y 2007 el número de defunciones por CM en Cataluña pasó de 1.085 a 956 y la tasa de mortalidad estandarizada por 100.000 mujeres varió de 34,8 a 23,3. La tabla 1 muestra las ratios de tasas (RT) de mortalidad en un modelo que incluye simultáneamente la edad, el periodo y la región. Se observa un descenso promedio del $3 \%$ anual $(\mathrm{RT}=0,97)$ en la tasa de mortalidad para el conjunto de Cataluña. A este ritmo, la tasa de mortalidad en el año 2007 se situó en torno al $65 \%$ de la tasa de 1993. La tabla 1 también permite comparar el comportamiento de las tasas de mortalidad por CM de las regiones sanitarias. Tomando como referencia Barcelona ciutat, se observaron a lo largo del período 1993-2007 tasas de mortalidad inferiores en las regiones Centre (RT=0,87), Costa de Ponent $(\mathrm{RT}=0,89)$, Tarragona $(\mathrm{RT}=0,9) \mathrm{y}$ Lleida $(\mathrm{RT}=0,92)$. El resto no mostró diferencias estadísticamente significativas respecto a Barcelona ciutat. Cuando se restringió el análisis al periodo 2000-2007 (datos no incluídos en la tabla 1), sólo la región Centre siguió mostrando una mortalidad menor, estadísticamente significativa, respecto a Barcelona ciutat.

Las diferencias detectadas al comparar las regiones con Barcelona ciutat no fueron lo suficientemente importantes como para resultar estadísticamente significativas al comparar cada región sanitaria (efectos aleatorios) con el conjunto de Cataluña (efectos fijos) mediante modelos lineales de efectos mixtos. No aparecieron diferencias estadísticamente significativas ni en la pendiente de las rectas de regresión (evolución de la mortalidad por CM) ni en los puntos de corte en el origen (nivel de mortalidad), tal como se aprecia en la figura 2.

La tendencia que siguieron las diferentes regiones en su descenso de las tasas de mortalidad fue uniforme, excepto en el caso de la región Centre. El análisis «joinpoint» solo mostró un cambio estadísticamente signifi- 


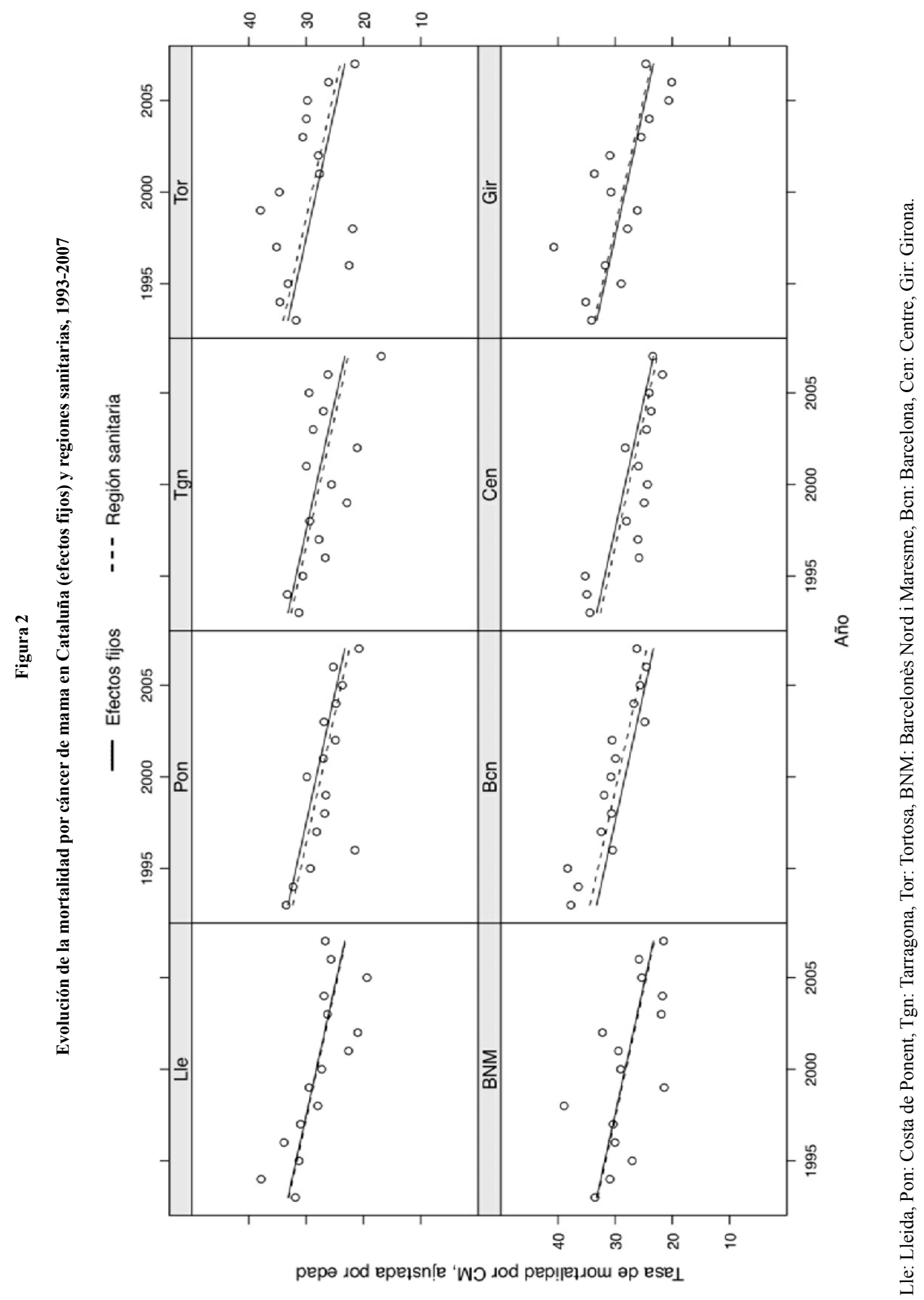




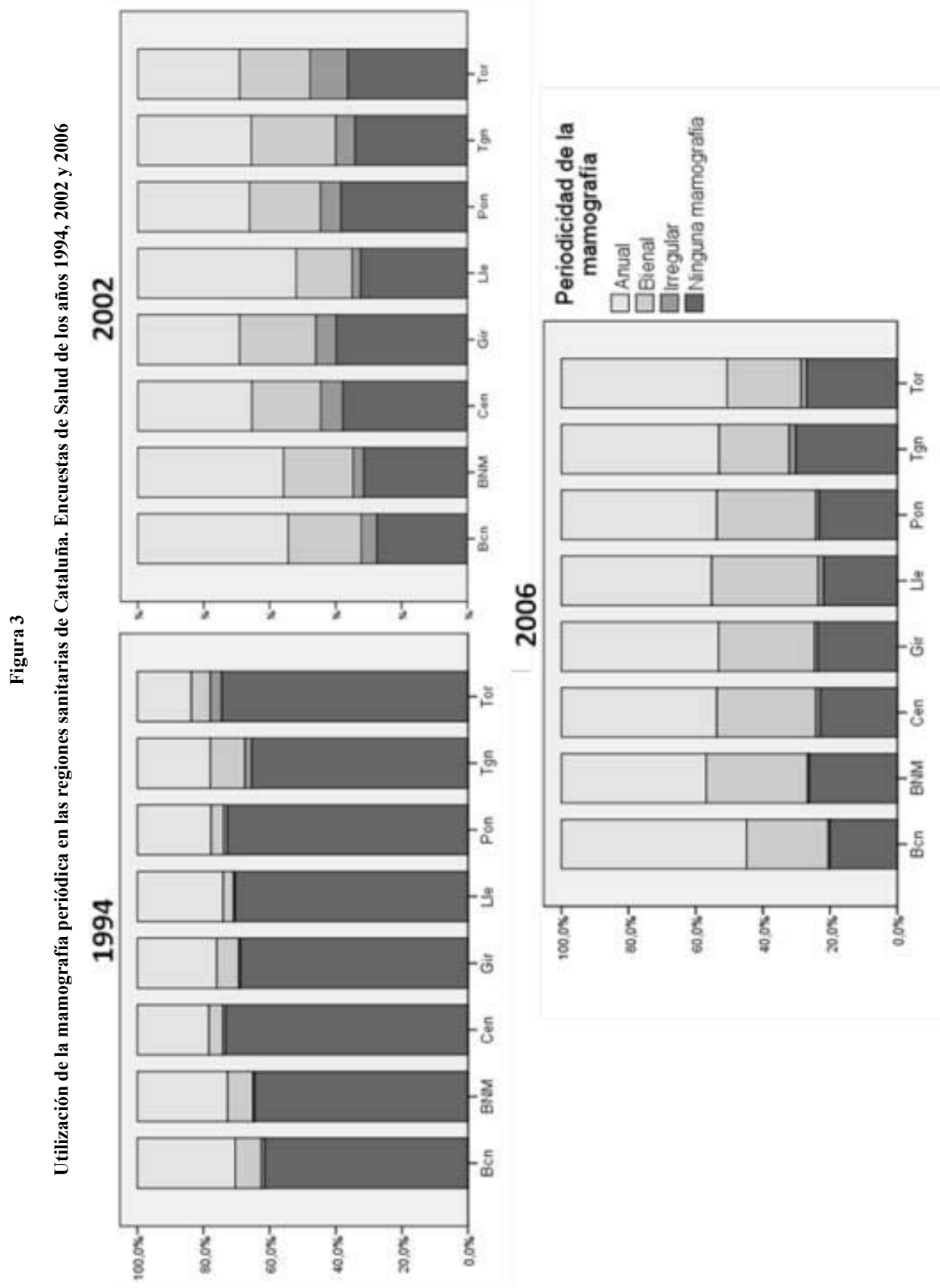

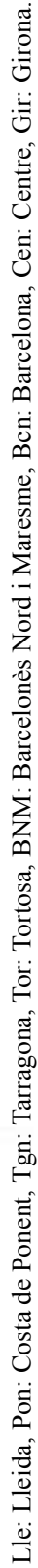

Rev Esp Salud Pública 2010, Vol. 84, N. ${ }^{\circ} 6$ 

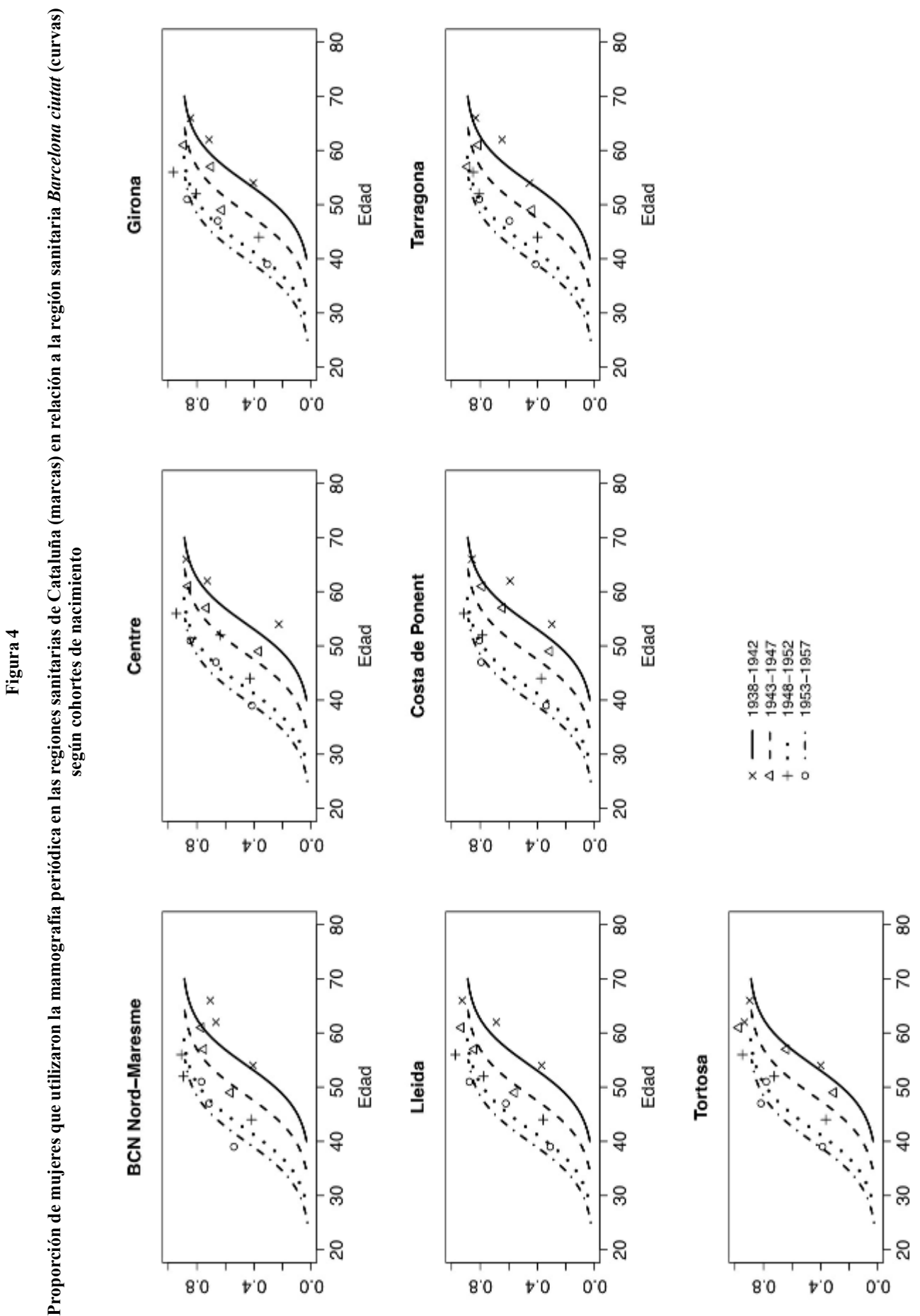


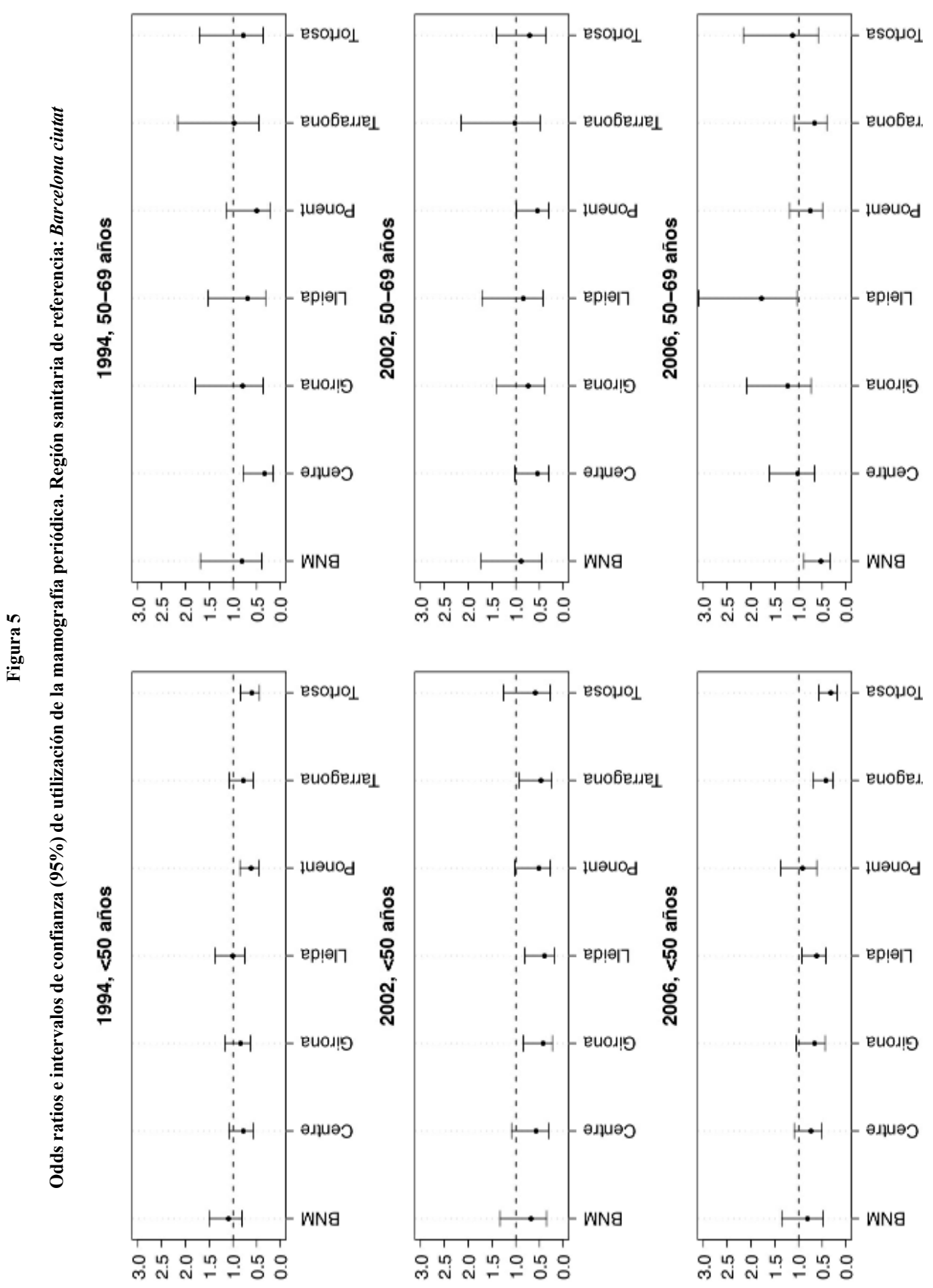


cativo en la tendencia de esa región, con un descenso muy marcado al principio (PCA igual a $-7,3[-14,3 ; 0,2])$ y más moderado a partir de 1997 (PCA igual a -1,5 [-3,4 ; 0,4]).

Diseminación de la mamografía periódica en Cataluña y regiones sanitarias. El porcentaje de mujeres entre 40 y 74 años que declararon hacerse mamografías periódicas pasó de $29,7 \%$ en 1994 a 64,0\% en 2002 y a $73,7 \%$ en 2006. El análisis descriptivo mostró diferencias en la utilización de la mamografía periódica según la región de residencia. En las tres encuestas de salud consecutivas un $24,1 \%$, un $54,3 \%$ y un $68,9 \%$ de las mujeres entre 40 y 74 afirmaban someterse a una mamografía periódica en la región de Tortosa, mientras que en Barcelona ciutat (la región donde se utilizó más la mamografía periódica en cualquier encuesta) las respuestas fueron afirmativas en un $36,1 \%$, un $69,9 \%$ y un $77,2 \%$, respectivamente (valor $\mathrm{p}$ de la prueba chi cuadrado $<0,001$ en las tres encuestas).

Por otro lado, la frecuencia con que las mujeres utilizaron la mamografía varió significativamente según la región (figura 3 ). Se observó una mayor realización de exámenes anuales en las regiones más pobladas y urbanas (valor $\mathrm{p}<0,01$ en las tres encuestas). Sólo la encuesta de 2006 recogió el motivo de la mamografía, y mostró que el Programa de Detección Precoz fue el impulsor de alrededor de un tercio de las mamografías realizadas periódicamente en Girona, Barcelona ciutat, Barcelonés Nord-Maresme y Centre. Lleida y Costa de Ponent superaron ligeramente las anteriores regiones y en Tortosa y Tarragona más de la mitad de las mujeres declararon hacerse las mamografías periódicas en el programa poblacional.

La figura 4 muestra la proporción de mujeres que utilizaron la mamografía periódica en las regiones sanitarias de Cataluña en relación a Barcelona ciutat, para las cohortes de mujeres nacidas en los periodos 1938$42,1943-47,1948-52$ y 1953-57. Se observa que, a cualquier edad, el uso de mamografía periódica va siendo mayor en las cohortes más recientes, fruto en gran parte de la implementación de los programas públicos de cribado. Los puntos o marcas corresponden a los resultados sobre el uso de mamografía periódica obtenidos de las encuestas para cada región y cohorte. Las regiones Centre y Costa de Ponent presentaron en algunos grupos de edad y cohortes porcentajes ligeramente inferiores a Barcelona ciutat (los puntos aparecen a la derecha de las curvas). Sin embargo, al contrastar la hipótesis de diferencias en los parámetros de las curvas de diseminación según regiones no se obtuvieron resultados estadísticamente significativos. Por lo tanto, el patrón seguido por las distintas regiones sanitarias en la incorporación de las mujeres a la utilización de la mamografía periódica es lo suficientemente similar como para no poder descartar esta hipótesis.

Al diferenciar por edades ( $<50$ versus 50 69 años) la utilización de la mamografía periódica en cada región sanitaria respecto a Barcelona ciutat se observan las odds ratios e intervalos de confianza (95\%) que muestra la figura 5. En mujeres de menos de 50 años se observó una utilización inferior y estadísticamente significativa de la mamografía periódica en Costa de Ponent y Tortosa el año 1994, Girona, Lleida y Tarragona el año 2002 y Lleida, Tarragona y Tortosa el año 2006. En mujeres entre 50 y 69 años se observaron utilizaciones inferiores a Barcelona ciutat, estadísticamente significativas o en el límite, en la región sanitaria Centre el año 1994, en Centre y Costa de Ponent el año 2002 y en Barcelonès Nord-Maresme el año 2006. Lleida mostró una utilización superior a Barcelona ciutat el año 2006.

\section{DISCUSIÓN}

Este trabajo se ha centrado en la comparación del nivel y la evolución de la mortalidad por CM, así como la utilización de la mamo- 
grafía periódica en las regiones sanitarias de Cataluña, en el periodo 1993-2007. En cuanto al nivel de las tasas de mortalidad por CM en las diferentes regiones sanitarias, al compararlas con el conjunto de Cataluña no se observaron diferencias estadísticamente significativas. Sin embargo, cuando se compararon las regiones con Barcelona ciutat, sí se observó un nivel inferior de mortalidad, estadísticamente significativo, en la regiones Centre, Costa de Ponent, Tarragona y Lleida. Este resultado es consistente con otros estudios que muestran mayor mortalidad por CM en las áreas urbanas o en mujeres con mayor nivel socioeconómico ${ }^{20}$. Por lo tanto, los resultados obtenidos para cada región se asemejan al conjunto pero, a la vez, presentan rasgos significativamente distintos cuando se comparan entre ellas. Además, el grado de similitud observado en el comportamiento de las distintas regiones no es independiente del período de análisis, obteniendo menores diferencias en los periodos más recientes, lo que indica que las tasas de mortalidad por CM de las regiones sanitarias han convergido.

La evolución de las tasas de mortalidad por CM en Cataluña ha seguido la misma tendencia observada en la mayoría de países occidentales desde la primera mitad de la década de 1990. La reducción de las tasas de mortalidad ha tenido un ritmo uniforme a lo largo del tiempo en todas las regiones, con la única excepción de la región Centre, que tuvo un descenso más marcado durante la primera mitad del periodo estudiado. Esto permite asumir que la tendencia decreciente observada para el conjunto de Cataluña a principios de los años noventa, antes de la puesta en marcha de los programas de cribado poblacional, y que ya ha sido señalada en otros estudios ${ }^{6,21}$, se produjo de forma homogénea en las regiones.

La utilización de la mamografía periódica en Cataluña es más frecuente en el grupo de mujeres que constituye la población diana del programa de cribado (50 a 69 años), aun- que en las tres encuestas analizadas es mayor la periodicidad anual que la bienal ${ }^{17}$, que es la establecida por dichos programas en Cataluña. Los resultados de nuestro trabajo no muestran grandes diferencias entre Barcelona ciutat y el resto de las regiones sanitarias en la utilización de la mamografía periódica. Sin embargo, al diferenciar dos grupos de edad ( $<50$ años y 50-69 años) algunas regiones sí presentaron variaciones significativas respecto a Barcelona ciutat. Estas diferencias no se han reflejado de forma clara en la evolución de la tasa de mortalidad. Por ejemplo, las mujeres de Barcelona ciutat utilizaron más la mamografía que las de otras regiones sanitarias y sin embargo tuvieron una mortalidad por cáncer de mama superior. En Tortosa encontramos una utilización inferior a la de Barcelona ciutat junto a una tasa de mortalidad bastante similar. La región Centre presentó simultáneamente la menor tasa de mortalidad y una baja utilización de la mamografía. Esta falta de asociación puede explicarse, en parte, por algunas limitaciones del estudio que impiden evaluar si ha habido una relación causal entre la utilización de la mamografía periódica y la evolución de la mortalidad por cáncer de mama. En primer lugar, se han analizado datos agregados, con el consiguiente riesgo de falacia ecológica. Una distribución más favorable del nivel socioeconómico en las mujeres residentes en Barcelona ciutat implicaría una utilización mayor de la mamografía. A la vez, características más prevalentes en zonas urbanas, como una menor fecundidad, maternidad a edad más avanzada, o mayor utilización de terapia hormonal substitutiva, se asocian a una mayor incidencia de $\mathrm{CM}^{22,23}$. Además, puesto que las regiones pueden presentar comportamientos distintos entre sí en función de si se estratifica por edad o no, o bien según el período de tiempo analizado, el análisis por grupos de edad quizá fuera más concluyente, pero la estratificación de los datos ocasionaría, en este estudio, problemas de estabilidad de las estimaciones. 
Por otro lado, existen limitaciones derivadas de la utilización de datos recogidos en encuesta, lo que presenta peligros de sesgo de memoria y error muestral. No obstante, al contrastarse los resultados de las encuestas de salud con otras fuentes de información (participación en el programa poblacional de detección precoz del CM u otras encuestas) se obtuvieron resultados consistentes ${ }^{17}$. Asimismo, para estimar la utilización de la mamografía se dispuso sólo de tres encuestas de salud poblacionales, con la consiguiente escasez de datos para las cohortes que nacieron a principios del siglo XX y después de 1960. De todos modos, estas cohortes no contribuyeron tanto al cálculo de la tasa de mortalidad ajustada como las cohortes intermedias. Finalmente, no hay que olvidar el efecto retardado de la detección precoz sobre la mortalidad, que no hemos podido estudiar.

La reciente reducción de la mortalidad por CM se atribuye a la introducción de la mamografía y a la mejora en los tratamientos ${ }^{24}$, aunque la cuantificación de su contribución está en revisión continua ${ }^{25}$. Por lo tanto, esperaríamos que desigualdades territoriales en el acceso a la mamografía o nuevos tratamientos se trasladaran en diferencias en la mortalidad entre regiones. En conclusión, la progresiva utilización de la mamografía por parte de las mujeres y la evolución de la mortalidad por CM fueron similares en las ocho regiones sanitarias de Cataluña. A pesar de que pueden encontrarse diferencias entre regiones, éstas disminuyeron a lo largo del tiempo. Por lo tanto, parece probable que la implantación de la mamografía en todo el territorio y la equidad en el acceso a tratamientos efectivos, contribuyeron a evitar desigualdades en la detección y mortalidad por cáncer de mama en Cataluña.

\section{AGRADECIMIENTOS}

Agradecemos a Pilar Brugulat, del Departament de Salut de la Generalitat de Catalu- ña, los datos de las encuestas de salud y la revisión del manuscrito y a las personas que evaluaron el trabajo durante el proceso editorial en la Revista Española de Salud Pública, cuyos comentarios contribuyeron a su mejora.

\section{BIBLIOGRAFÍA}

1. Ferlay J, Autier P, Boniol M, Heanue M, Colombet M, Boyle P. Estimates of the cancer incidence and mortality in Europe in 2006. Ann Oncol. 2007;18(3):581-92.

2. Berry DA, Cronin KA, Plevritis SK, Fryback DG, Clarke L, Zelen M, et al. Effect of screening and adjuvant therapy on mortality from breast cancer. $\mathrm{N}$ Engl J Med. 2005;353(17):1784-92.

3. Gispert R, Cleries R, Puigdefabregas A, Freitas A, Esteban L, Ribes J. Tendencia de la mortalidad por cáncer en Cataluña, 1985-2004. Med Clin (Barc). 2008;131 Suppl 1:25-31.

4. Puig X, López-Abente G, Gispert R, Freitas A, Puigdefàbregas A. Tendències de la mortalitat a Catalunya, 1978-2002. Models edat/període/ cohort. Barcelona: Departament de Salut. Generalitat de Catalunya, 2005. Disponible en: http://www. gencat.cat/salut/depsalut/pdf/t1978-2002.pdf.

5. Puig X, Ginebra J, Gispert R. Análisis de la evolución temporal de la mortalidad mediante modelos lineales generalizados. Gac Sanit. 2005;19(6):481-5.

6. Izquierdo A, Gispert R, Saladie F, Espinas JA. Análisis de la incidencia, la supervivencia y la mortalidad según las principales localizaciones tumorales, 1985-2019: Cáncer de mama. Med Clin (Barc). 2008;131 Suppl 1:50-2.

7. Departament de Salut. Programa de detecció precoç de càncer de mama de Catalunya. Situació actual i perspectives de futur. 2006. Barcelona: Direcció General de Planificació i Avaluació. Disponible en: http://www.gencat.cat/salut/depsalut/ pdf/informecacc2006.pdf.

8. Masuet C, Seculi E, Brugulat P, Tresserras R. La práctica de la mamografía preventiva en Cataluña. Un paso adelante. Gac Sanit. 2004;18(4):321-5.

9. Rohlfs I, Borrell C, Plasencia A, Pasarin I. Social inequalities and realisation of opportunistic screening mammographies in Barcelona (Spain). J Epidemiol Community Health. 1998;52(3):205-6. 
10. Padró continu. Institut d'Estadística de Catalunya (IDESCAT). 2009. Disponible en: http://www. idescat.cat/cat/poblacio/poblrecomptes.html.

11. Servei Català de la Salut. Els catalans parlen de la seva salut. Enquesta de salut de Catalunya 1994. Barcelona: Generalitat de Catalunya, Departament de Sanitat i Seguretat Social; 1998. Disponible en: http://www.gencat.cat/salut/depsalut/html/ca/plasalut/ensalut1994.htm.

12. Direcció General de Salut Pública. La salut i els serveis sanitaris a Catalunya. La visió dels ciutadans 1'any 2002. Barcelona: Generalitat de Catalunya. Departament de Sanitat i Seguretat Social; 2003. Disponible en: http://www.gencat.cat/ salut/depsalut/html/ca/plasalut/doc5365.html.

13. Departament de Salut. Enquesta de Salut de Catalunya 2006. Els catalans parlen de la seva salut i dels serveis sanitaris. Barcelona: Direcció General de Planificació i Avaluació, Departament de Salut, Generalitat de Catalunya ; 2009. Disponible en: http://www.gencat.cat/salut/depsalut/html/ca/plasalut/catparsesalut2006.pdf y http://www.gencat. cat/salut/depsalut/html/ca/plasalut/doc32868.html.

14. Agresti A. Categorical data analysis. New York: Wiley and Sons; 1990.

15. Surveillance Research Program, US National Cancer Institute. Joinpoint.

16. Pinheiro JC, Bates DM. Mixed-effects models in S and S-PLUS. New York: Springer-Verlag; 2000.

17. Rue M, Carles M, Vilaprinyo E, Martinez-Alonso M, Espinas JA, Pla R, et al. Dissemination of periodic mammography and patterns of use, by birth cohort, in Catalonia (Spain). BMC Cancer. 2008; 8:336.
18. Stata Corporation. Stata/SE. 2009; 11.0 for Macintosh.

19. The R Foundation for Statistical Computing. R. $2009 ; 2.10 .1$.

20. Pollan M, Ramis R, Aragones N, Perez-Gomez B, Gomez D, Lope V, et al. Municipal distribution of breast cancer mortality among women in Spain. BMC Cancer. 2007;7:78.

21. Vidal-Lancis C, Martínez-Sánchez JM, MateosMazón M., Peris-Tuser M. Mortalidad por cáncer de mama: Evolución en España y sus comunidades autónomas durante el periodo 1980-2005. Rev Esp Salud Pública. 2010;84:53-9.

22. Kelsey JL, Gammon MD, John EM. Reproductive factors and breast cancer. Epidemiol Rev. 1993;15(1):36-47.

23. Hausauer AK, Keegan TH, Chang ET, Glaser SL, Howe H, Clarke CA. Recent trends in breast cancer incidence in US white women by county-level urban/rural and poverty status. BMC Med. 2009;7:31.

24. Cronin KA, Feuer EJ, Clarke LD, Plevritis SK. Impact of adjuvant therapy and mammography on U.S. mortality from 1975 to 2000: Comparison of mortality results from the CISNET breast cancer base case analysis. J Natl Cancer Inst Monogr. 2006; (36):112-21.

25. Kalager M, Zelen M, Langmark F, Adami HO. Effect of screening mammography on breast-cancer mortality in Norway. N Engl J Med. 2010;363 (13):1203-10. 